\title{
Efficacy and Safety of Endovascular Treatment in Patients with Internal Carotid Artery Occlusion and Collateral Middle Cerebral Artery Flow
}

\author{
Yong-Won Kim, M.D., ${ }^{1-3}$ Dong-Hun Kang, M.D., ${ }^{2-4}$ Yong-Sun Kim, M.D., ${ }^{2,3}$ Yang-Ha Hwang, M.D., \\ Departments of Neurology, Radiology, ${ }^{2}$ School of Medicine, Kyungpook National University, Daegu, Korea \\ Cerebrovascular Center, Kyungpook National University Hospital, Daegu, Korea \\ Department of Neurosurgery, ${ }^{4}$ School of Medicine, Kyungpook National University, Daegu, Korea
}

Objective : In patients with internal carotid artery (ICA) occlusion, collateral middle cerebral artery (MCA) flow has a protective role against ischemia. However, some of these patients may experience initial major neurological deficits and major worsening on following days. Thus, we investigated the safety and efficacy of endovascular treatment (EVT) for ICA occlusion with collateral MCA flow by comparing clinical outcomes of medical treatment versus EVT.

Methods : The inclusion criteria were as follows : 1) acute ischemic stroke with ICA occlusion and presence of collateral MCA flow on transfemoral cerebral angiography (TFCA) and 2) hospital arrival within 12 hours from symptom onset. The treatment strategy was made by the attending physician based on the patient's clinical status and results of TFCA.

Results : Eighty-one patients were included (30 medical treatment, $51 \mathrm{EVT}$ ). The EVT group revealed a high incidence of intracranial ICA occlusion, longer ipsilesional MCA contrast filling time, and a similar rate of favorable clinical outcome despite a higher mean baseline the National Institutes of Health Stroke Scale (NIHSS) score. By binary logistic regression analysis, intravenous recombinant tissue plasminogen activator and EVT were independent predictors of favorable clinical outcome. In subgroup analysis based on stroke etiology, the non-atherosclerotic group showed a higher baseline NIHSS score, higher incidence of EVT, and a higher rate of distal embolization during EVT in comparison with the atherosclerotic group.

Conclusion : In patients with ICA occlusion and collateral MCA flow, decisions regarding treatment strategy based on TFCA can help achieve favorable clinical outcomes. EVT strategy with respect to etiology of ICA occlusion might help achieve better angiographic outcomes.

Key Words : Stroke · Internal carotid artery · Collateral circulation · Thrombectomy.

\section{INTRODUCTION}

Acute ischemic stroke due to internal carotid artery (ICA) occlusion generally has a poor clinical outcome ${ }^{19)}$. However, clinical outcomes can vary according to the extent of ischemia, which in turn can be associated with the location and

- Received : June 29, 2018 •Revised : August 12, 2018 •Accepted : September 5, 2018

- Address for reprints : Yang-Ha Hwang, M.D.

Department of Neurology, School of Medicine, Kyungpook National University, 680 Gukchaebosang-ro, Jung-gu, Daegu 41944, Korea

Tel : +82-53-420-5765, Fax : +82-53-422-4265, E-mail : yangha.hwang@gmail.com

This is an Open Access article distributed under the terms of the Creative Commons Attribution Non-Commercial License (http://creativecommons.org/licenses/by-nc/4.0) which permits unrestricted non-commercial use, distribution, and reproduction in any medium, provided the original work is properly cited. 
shape of the occlusive ICA and the collateral strength ${ }^{14,18)}$. Specifically, the presence of collateral middle cerebral artery (MCA) flow via the circle of Willis is associated with a protective role against cerebral ischemia; and therefore, acute stroke attributable to ICA occlusion with collateral MCA flow can generally show mild neurological deficits at onset and relatively favorable clinical outcomes ${ }^{4,13,14,16)}$. However, in this situation, about $50 \%$ of patients with mild to moderate neurological severity at baseline experience major worsening on following days and about $25 \%$ of patients present with initial major neurological deficits ${ }^{13)}$.

Despite recent successful clinical trials of endovascular treatment (EVT) for anterior circulation stroke ${ }^{3,6,7,11,21)}$, the evidence regarding EVT for ICA occlusion with collateral MCA flow is still lacking. But theoretically, it can be presumed that EVT for ICA occlusion with collateral MCA flow would be difficult due to the long length of occlusion, a large clot burden, and the risk of complications, such as distal embolization $^{2,15)}$. Therefore, the clinician may hesitate to perform EVT due to the aforementioned reasons; rather, the best medical treatment for such patients may be preferred. Therefore, we investigated the safety and efficacy of EVT for ICA occlusion with collateral MCA flow by comparing clinical outcomes between a medical treatment group and an EVT group, and we tried to find good candidates who will benefit from EVT.

\section{MATERIALS AND METHODS}

\section{Patients}

This study is a retrospective review from a consecutively collected acute ischemic stroke registry at Kyungpook National University Hospital between December 2006 and May 2017. The inclusion criteria for this study were as follows : 1) acute ischemic stroke with symptomatic ICA occlusion, 2) hospital arrival within 12 hours from symptom onset, 3) presence of collateral MCA flow on transfemoral cerebral angiography (TFCA), and 4) pre-stroke modified Rankin Scale (mRS) score $\leq 2$. Patients with other stroke etiologies, including Moyamoya disease and vasculitis, were excluded. This study was approved by the local Insitutional Review Board of Kyungpook National University Hospital (KNUH 2015-02022).

\section{Baseline assessment and endovascular procedure}

After initial neurological examination, non-enhanced brain computed tomography (CT) and CT angiography were performed. Intravenous recombinant tissue plasminogen activator (rtPA) was administered in eligible patients based on the guidelines for the early management of acute ischemic stroke $^{10)}$. In some cases, such as a late time window, brain magnetic resonance (MR) imaging and $\mathrm{MR}$ angiography were performed.

All included patients had TFCA and completed angiographic evaluation, including bilateral carotid and vertebral angiogram for assessment of vascular occlusion and collateral status. A 5 F guide catheter (Headhunter, Cordis, Miami, FL, USA) was used for the cerebral angiogram, and $10 \mathrm{~mL}$ of iodinate contrast was administered at $3 \mathrm{~mL} / \mathrm{sec}$. To evaluate the collateral circulation, we assessed the contrast filling time of the arterial phase from the ipsilesional and contralateral MCA territory. For collateral flow via the anterior communicating artery, a contralateral carotid angiogram (distal common carotid artery [CCA] or proximal ICA) was performed. In the other collateral route, two angiogram series (contralateral CCA and similar level of V2 segment of the dominant vertebral artery in collateral flow via posterior communicating artery, bilateral distal CCA in collateral flow via the ipsilesional ophthalmic artery) were used. The decision for EVT was made according to the attending physician's discretion based on the patient's clinical status and the results of TFCA. According to the institutional EVT protocol, contact aspiration thrombectomy was performed in most cases. If recanalization was failed after three attempts, we used a stent retriever. However, a stent retriever was used preferentially instead of an aspiration catheter in cases of difficult target access due to high vascular tortuosity or heavy calcification. Carotid balloon angioplasty or stenting was conducted in cases of extracranial ICA severe stenosis with distal flow compromise

\section{Clinical and radiographic evaluation}

Data, including demographics, neurological status, and angiography findings, were collected from the patients' record. The National Institute of Health Stroke Scale (NIHSS) score was assessed at baseline, day 1, and day 7 (or at discharge if it was before day 7). Early neurological deterioration (END) was defined as an increase in NIHSS score of 4 points or more that persists for at least 24 hours within 7 days. Early neurological 
improvement (ENI) was defined as a decrease in NIHSS score of 4 points or more 24 hours after treatment. The Alberta Stroke Program Early CT Score (ASPECTS) was calculated from the initial diffusion-weighted image (DWI). The clinical outcomes were assessed using the mRS at 3 months, and a favorable clinical outcome was defined as an $\mathrm{mRS}$ score of $\leq 2$. Reperfusion status was measured using the modified Thrombolysis In Cerebral Infarction (mTICI) score ${ }^{23)}$. The mTICI score was independently evaluated by an experienced neuroradiologist and neurologist who were blinded to the clinical information. If there was a discrepancy, the final assessment was determined by consensus. Successful reperfusion was defined as an mTICI score of $2 \mathrm{~b}$ or 3 .

A follow-up brain CT scan was acquired immediately and 1 day after the EVT. The type of intracranial hemorrhage was determined according to the European Cooperative Acute Stroke Study II classification system ${ }^{9)}$. Symptomatic intracranial hemorrhage was defined as any type of hemorrhage with an increase of $\geq 4$ in NIHSS score within 24 hours $^{22)}$.

\section{Statistics}

Statistical analyses were performed using SPSS version 22.0 (IBM, Armonk, NY, USA). Chi-squared or Fisher's exact tests were used for categorical data. The Student's t or Mann-Whitney $U$ tests were used for continuous variables. Binary logistic regression analysis was performed to identify independent predictors of favorable clinical outcome at 3 months. Independent variables with a $p$ value less than 0.10 in univariate analysis for favorable clinical outcome were put into a binary logistic regression analysis. EVT related to the aim of this study was put into the binary logistic regression analysis, though it was not significant in the univariate analysis. A $p$ value $<0.05$ was considered statistically significant.

\section{RESULTS}

Eighty-one patients who had an ICA occlusion with collateral MCA flow were included in this study. Thirty patients received medical treatment and 51 patients received EVT with medical treatment.

\section{Baseline characteristics}

Baseline characteristics, including demographics and risk
Table 1. Baseline characteristics

\begin{tabular}{|c|c|c|c|}
\hline Variable & $\begin{array}{l}\text { Medical } \\
\text { treatment } \\
(n=30)\end{array}$ & $\operatorname{EVT}(n=51)$ & $p$-value \\
\hline Age & $68(61.75-78.25)$ & $73(64-78)$ & $0.252^{*}$ \\
\hline Female & $8(26.7)$ & $22(43.1)$ & 0.138 \\
\hline Intravenous rtPA use & $9(30.0)$ & $20(39.2)$ & 0.403 \\
\hline Baseline NIHSS & $13(9-17.25)$ & $16(11-20)$ & $0.074^{*}$ \\
\hline Onset to door time & 231.5 (89-382) & $268(113-693.5)$ & $0.315^{*}$ \\
\hline ASPECTS DWI & $9(8-10)$ & $9(8-10)$ & $0.700^{*}$ \\
\hline \multicolumn{4}{|l|}{ Medical history } \\
\hline Hypertension & $21(70.0)$ & $33(64.7)$ & 0.625 \\
\hline Diabetes & $12(40.0)$ & $18(35.3)$ & 0.672 \\
\hline Hyperlipidemia & $18(60.0)$ & $18(35.3)$ & 0.031 \\
\hline Atrial fibrillation & $9(30.0)$ & $22(43.1)$ & 0.24 \\
\hline Coronary disease & $6(20.0)$ & $8(15.7)$ & 0.62 \\
\hline Current smoking & $11(36.7)$ & $20(39.2)$ & 0.82 \\
\hline Previous Stroke & $5(16.7)$ & $9(17.6)$ & 0.91 \\
\hline Antiplatelet use & $9(30.0)$ & $22(43.1)$ & 0.24 \\
\hline Anticoagulant use & $3(10.0)$ & $2(3.9)$ & $0.354^{*}$ \\
\hline Occlusion location & & & 0.005 \\
\hline Extracranial ICA & $20(66.7)$ & $15(29.4)$ & \\
\hline Intracranial ICA & $8(26.7)$ & $27(52.9)$ & \\
\hline $\begin{array}{l}\text { ICA and tandem MCA } \\
\text { branches }\end{array}$ & $2(6.7)$ & $9(17.6)$ & \\
\hline Route of collaterals & & & 0.676 \\
\hline $\begin{array}{l}\text { Anterior communicating } \\
\text { artery }\end{array}$ & $15(50.0)$ & $22(43.1)$ & \\
\hline $\begin{array}{l}\text { Posterior communicating } \\
\text { artery }\end{array}$ & $8(26.7)$ & $12(23.5)$ & \\
\hline $\begin{array}{l}\text { Anterior \& posterior } \\
\text { communicating artery }\end{array}$ & $2(6.7)$ & $8(15.7)$ & \\
\hline Ophthalmic artery & $5(16.7)$ & $9(17.6)$ & \\
\hline $\begin{array}{l}\text { Delay of contrast filling time } \\
\text { for MCA territory (seconds) }\end{array}$ & $1.5(1.0-2.1)$ & $3.0(2.2-4.0)$ & $<0.001$ \\
\hline Stroke subtypes & & & 0.024 \\
\hline Large artery atherosclerosis & $21(70.0)$ & $21(41.2)$ & \\
\hline Cardioembolism & $5(16.7)$ & $23(45.1)$ & \\
\hline Undetermined & $4(13.3)$ & $7(13.7)$ & \\
\hline
\end{tabular}

Values are presented as median (interquartile range) or number (\%). *Mann whitney U test. EVT : endovascular treatment, rtPA : recombinant tissue plasminogen activator, NIHSS : National Institute of Health Stroke Scale, ASPECTS : Alberta Stroke Program Early CT Score, DWI : diffusionweighted imaging, ICA : internal carotid artery, MCA : middle cerebral artery 
factors, were summarized in Table 1. The median age was 68 (interquartile range [IQR], 61.75-78.25) years in the medical treatment group and 73 (IQR, 64-78) years in the EVT group $(p=0.252)$. Intravenous rtPA was administered in nine patients $(30 \%)$ in the medical treatment group and in 20 patients (39.2\%) in the EVT group ( $p=0.403$ ). The DWI ASPECTS was similar between groups ( 9 [IQR, 8-10] vs. 9 [IQR, 8-10], $p=0.700$ ), but the baseline NIHSS score was higher in the EVT group, though not statistically significant (13 vs. $16, p=0.074$ ). The incidence of hyperlipidemia was higher in the medical treatment group (60.0\% vs. $35.3 \%, p=0.031)$, and other risk factors for stroke were not statistically different between groups. Regarding stroke etiology, the medical treatment group showed a higher proportion of large artery atherosclerosis than the EVT group. Median time (in seconds) for ipsilesional MCA contrast filling during the arterial phase was significantly longer in the EVT group (1.5 [IQR, 1.0-2.1] vs. 3.0 [IQR, 2.2-4.0], $p=0.024$ ).

\section{Angiographic and clinical outcomes between medical treatment and EVT groups}

Clinical outcomes of both groups are summarized in Table 2. The median NIHSS score measured 1 day after stroke onset was 10 (IQR, 5.75-14) in the medical treatment group and 12 (IQR, 6-18) in the EVT group ( $p=0.172)$, and the median score measured 7 days after stroke onset was 8.5 (IQR, 3.75$14)$ in the medical treatment group and 8 (IQR, 2-15) in the EVT group ( $p=0.941$ ). The EVT group revealed a lower incidence of END and a higher incidence of ENI, but the differ-

Table 2. Details of clinical outcomes

\begin{tabular}{lccc}
\hline Variable & $\begin{array}{c}\text { Medical treatment } \\
(\mathbf{n = 3 0 )}\end{array}$ & EVT (n=51) & $\boldsymbol{p}$-value \\
\hline NIHSS 1 day & $10(5.75-14)$ & $12(6-18)$ & $0.172^{*}$ \\
NIHSS 7 day & $8.5(3.75-14)$ & $8(2-15)$ & $0.941^{*}$ \\
$\begin{array}{l}\text { Early neurological } \\
\text { deterioration }\end{array}$ & $7(23.3)$ & $6(11.8)$ & $0.215^{\dagger}$ \\
$\begin{array}{l}\text { Early neurological } \\
\text { improvement }\end{array}$ & $15(50.0)$ & $32(62.7)$ & 0.262 \\
mRS 0-2 at 3 months & $13(43.3)$ & $27(52.9)$ & 0.404 \\
Mortality & $4(13.3)$ & $4(7.8)$ & $0.460^{\dagger}$ \\
\hline
\end{tabular}

Values are presented as median (interquartile range) or number (\%). *Mann whitney U test. ${ }^{\dagger}$ Fisher's exact T test. EVT : endovascular treatment, NIHSS : National Institute of Health Stroke Scale, mRS : modified Rankin Scale ences were not statistically significant. The rates of favorable clinical outcome at 3 months were $43.3 \%$ of patients in the medical treatment group and $52.9 \%$ of patients in the EVT group ( $p=0.404$ ), and the mortality rate was not statistically different between groups ( $13.3 \%$ vs. $7.8 \%, p=0.460)$. By binary logistic regression analysis, intravenous $\operatorname{rtPA}(p=0.006)$ and EVT ( $p=0.041)$ were independent predictors of a favorable clinical outcome. And atherosclerotic ICA occlusion $(p=0.084)$ showed a tendency for a favorable clinical outcome (Table 3).

Subgroup analysis of angiographic and clinical outcomes according to treatment strategy and etiology of ICA occlusion

Baseline characteristics and clinical outcomes according to treatment strategy for each ICA occlusion etiology are summarized in Table 4. The overall non-atherosclerotic ICA occlusion group showed a higher baseline NIHSS score (13 vs. $16.5, p=0.011)$ and EVT was more commonly applied $(51.2 \%$ vs. $76.3 \%, p=0.019)$. The median NIHSS score at the baseline and day 1 was significantly higher in the non-atherosclerotic ICA occlusion group, but became similar between groups at day 7. The favorable clinical outcome at 3 months was not statistically different between groups (58.1\% vs. 39.5\%, $p=0.094$ ). In comparison between treatment strategies within each etiology, the EVT group revealed a higher baseline NIHSS score (11 vs. $16.5, p=0.027)$ and similar clinical outcomes in the atherosclerotic ICA occlusion group. In the non-atherosclerotic ICA occlusion group, the EVT group achieved a higher rate of favorable clinical outcome, but the result was not statistically significant ( $22.2 \%$ vs. $44.8 \%, p=0.273$ ).

Angiographic outcomes of the patients who received EVT were summarized in Table 5. The atherosclerotic ICA occlusion group revealed a higher rate of mTICI $2 b$ or 3 of reperfusion, but the result was not statistically significant ( $90.9 \%$ vs.

Table 3. Binary logistic regression analysis for favorable clinical outcome at 3 months

\begin{tabular}{lcc}
\hline Variable & Odds ratio $(95 \% \mathrm{Cl})$ & $\boldsymbol{p}$-value \\
\hline Age (/1 year) & $0.956(0.901-1.013)$ & 0.128 \\
Sex (female) & $0.502(0.154-1.641)$ & 0.254 \\
Intravenous rtPA & $5.367(1.609-17.907)$ & 0.006 \\
Baseline NIHSS & $0.909(0.816-1.012)$ & 0.081 \\
Endovascular treatment & $3.452(1.054-11.304)$ & 0.041 \\
Atherosclerotic occlusion & $2.837(0.868-9.268)$ & 0.084 \\
\hline
\end{tabular}

$\mathrm{Cl}$ : confidence interval, rtPA : recombinant tissue plasminogen activator, NIHSS : National Institute of Health Stroke Scale 
$75.9 \%, p=0.268$ ). The time from groin puncture to achieving final reperfusion was 64.5 minutes (IQR, 46.5-79.25) minutes in the atherosclerotic occlusion group and 69 minutes (IQR, 44-141) minutes in the non-atherosclerotic occlusion group $(p=0.430)$. But, the distal embolization rate into the down- stream of the occluded ICA during the EVT procedure was significantly higher in the non-atherosclerotic occlusion group ( 0 vs. $58.6 \%, p<0.001$ ). Symptomatic intracerebral hemorrhage did not occur in either group.

Table 4. Baseline characteristic and clinical outcomes according to treatment strategy of each etiology of ICA occlusion

\begin{tabular}{|c|c|c|c|c|c|c|c|c|c|}
\hline \multirow[b]{2}{*}{ Variable } & \multicolumn{4}{|c|}{ Atherosclerotic ICA occlusion group $(n=43)$} & \multicolumn{4}{|c|}{ Non-atherosclerotic ICA occlusion group $(n=38)$} & \multirow[b]{2}{*}{$p$-value } \\
\hline & Overall & $\begin{array}{l}\text { Medical } \\
\text { Treatment } \\
(n=21)\end{array}$ & $\operatorname{EVT}(n=22)$ & $p$-value & Overall & $\begin{array}{c}\text { Medical } \\
\text { Treatment } \\
(n=9)\end{array}$ & EVT $(n=29)$ & $p$-value & \\
\hline Baseline NIHSS & $13(9-19)$ & $11(7.5-14.5)$ & $16.5(10-20)$ & $0.027^{*}$ & $16.5(13.75-20.25)$ & $18(14.5-22)$ & $16(12-20)$ & $0.276^{*}$ & $0.011^{*}$ \\
\hline ASPECTS DWI & $9(7-10)$ & $9(8-10)$ & $8(7-10)$ & $0.148^{*}$ & $9(8-10)$ & $9(8-9.5)$ & $9(9-10)$ & $0.248^{*}$ & $0.143^{*}$ \\
\hline NIHSS 1 day & $9(5-13)$ & $7(3-12)$ & $11(6.75-15.5)$ & $0.051^{*}$ & $14(10.5-18.0)$ & $14(12.5-17)$ & $14(5.5-18)$ & $0.667^{*}$ & $0.015^{*}$ \\
\hline NIHSS 7 day & $7(3-13)$ & $8(3-12.5)$ & $7(2.75-15.25)$ & $0.922^{*}$ & $10(5.5-15.5)$ & $13(6.5-21.5)$ & $10(2-15)$ & $0.524^{*}$ & $0.195^{*}$ \\
\hline END & $7(16.3)$ & $5(23.8)$ & $2(9.1)$ & $0.240^{\dagger}$ & $6(15.8)$ & $2(22.2)$ & $4(13.8)$ & $<0.613^{\dagger}$ & 0.952 \\
\hline ENI & $24(55.8)$ & $10(47.6)$ & $14(63.6)$ & $0.364^{\dagger}$ & $23(60.5)$ & $5(55.6)$ & $18(62.1)$ & $<0.999^{\dagger}$ & 0.668 \\
\hline mRS $0-2$ at 3 months & $25(58.1)$ & $11(52.4)$ & $14(63.6)$ & 0.455 & $15(39.5)$ & $2(22.2)$ & $13(44.8)$ & $0.273^{\dagger}$ & 0.094 \\
\hline Mortality & $4(9.3)$ & $3(14.3)$ & $1(4.5)$ & $0.345^{\dagger}$ & $4(10.5)$ & $1(11.1)$ & $3(10.3)$ & $<0.999^{\dagger}$ & $>0.999^{\dagger}$ \\
\hline
\end{tabular}

Values are presented as median (interquartile range) or number (\%). *Mann whitney U test. ${ }^{\dagger}$ Fischer's exact T test. ICA : internal carotid artery, EVT : endovascular treatment, NIHSS : National Institute of Health Stroke Scale, ASPECTS : Alberta Stroke Program Early CT score, DWI : diffusion-weighted imaging, END : early neurological deterioration, ENI : early neurological improvement, mRS : modified Rankin Scale

Table 5. Angiographic outcomes according to the etiology of ICA occlusion

\begin{tabular}{|c|c|c|c|}
\hline Variable & $\begin{array}{l}\text { Atherosclerotic occlusion } \\
\qquad(n=22)\end{array}$ & $\begin{array}{l}\text { Non-atherosclerotic occlusion } \\
\qquad(n=29)\end{array}$ & $p$-value \\
\hline Door to puncture time (minutes) & $94(87.25-116.25)$ & $82(69-103)$ & 0.058 \\
\hline Groin puncture to final reperfusion time (minutes) & $64.5(46.5-79.25)$ & $69(44-141)$ & 0.430 \\
\hline EVT procedure time (minutes) & $44.5(19.75-56.25)$ & $47(21-116)$ & 0.274 \\
\hline Onset to final reperfusion time (minutes) & $459(263.75-1008.25)$ & $444.5(306-702.25)$ & 0.755 \\
\hline EVT strategy & & & $<0.001$ \\
\hline MT only & $11(50.0)$ & $29(100.0)$ & \\
\hline MT \& carotid stenting & $9(40.9)$ & 0 & \\
\hline MT \& balloon angioplasty & $2(9.1)$ & 0 & \\
\hline $\mathrm{mTICl} 2 \mathrm{~b}-3$ of reperfusion & $20(90.9)$ & $22(75.9)$ & $0.268^{*}$ \\
\hline Distal embolization & 0 & $17(58.6)$ & $<0.001$ \\
\hline Hemorrhagic complications & & & 0.454 \\
\hline Intraventricular hemorrhage & 0 & $1(3.4)$ & \\
\hline Parenchymal hematoma & 0 & 0 & \\
\hline Subarachnoid hemorrhage & 0 & $1(3.4)$ & \\
\hline Symptomatic ICH & 0 & 0 & \\
\hline mRS $0-2$ at 3 months & 14 (63.6) & $13(44.8)$ & 0.183 \\
\hline
\end{tabular}

Values are presented as median (interquartile range) or number (\%). *Fischer's exact T test. ICA : internal carotid artery, EVT : endovascular treatment, MT : mechanical thrombectomy, $\mathrm{mTICI}$ : modified Treatment in Cerebral Infarction, ICH : intracerebral hemorrhage, mRS : modified Rankin Scale 


\section{DISCUSSION}

The exact mechanism of a developing ICA occlusion with collateral MCA flow is still unknown. We suppose two possible mechanisms. First, when antegrade ICA flow diminishes due to carotid stenosis or a thrombus, collateral channels via the Willisian route are opened. If the collateral flow is sufficient, retrograde ICA flow from collateral flow could prevent further distal migration of the blood clot and promote coagulation within the ICA by stasis of arterial flow. Second, complete arrest of ICA flow caused by a large burden of clotted blood or underlying atherosclerosis can also make an ICA occlusion with collateral MCA flow. Either way, a large amount of blood clot within the occluded ICA can be expected.

When considering EVT, it is definitely a difficult procedure due to large clot burden or underlying stenosis, and the possibility of distal embolization during EVT is also high ${ }^{2,5}$. Also, considering medical therapy, clinical deterioration is possible due to diminished collateral flow and/or distal embolization due to clot fragmentation/migration. Therefore, the clinician cannot help but wonder whether EVT or medical treatment is better for patients with an ICA occlusion with collateral MCA flow.

The main finding of this study was that EVT demonstrated reasonable clinical outcomes in patients with an ICA occlusion with collateral MCA flow. Moreover, favorable clinical outcome was affected by administration of intravenous rtPA and EVT. Further analysis regarding etiology of the ICA occlusion was as follows : 1) In the atherosclerotic ICA occlusion group, both medical treatment and EVT groups showed a high rate of favorable clinical outcome, and EVT seemed to be safe and 2) In the non-atherosclerotic occlusion group, the clinical outcome for the medical treatment group was dismal, and the EVT group revealed a high rate of distal embolization during the procedure despite a relatively higher favorable clinical outcome ( $22.2 \%$ vs. $44.8 \%, p=0.273)$.

The clinical course of patients with ICA occlusion with collateral MCA flow is still unknown due to a lack of large clinical trials. However, Kim et al. ${ }^{13)}$ reported the clinical outcome of 24 medically treated patients with ICA occlusion and collateral MCA flow. The patients in that report had mild neurological deficits (median baseline NIHSS score of 6); 33.3\% of those patients had END, and $41.7 \%$ had an mRS $0-2$ at 3 months ${ }^{13)}$. However, this study demonstrated a lower inci- dence of END and a higher incidence of favorable clinical outcomes regardless of treatment strategy. The authors speculated that the better outcome in this study was associated with the decision of treatment strategy based on the assessment of collateral status by the TFCA.

The collateral circulation has a protective role for ischemic brain injury and would be recruited to preserve cerebral perfusion to compensate for the effects of ischemia after arterial occlusion $^{4,16)}$. Collateral flow via the circle of Willis maintains cerebral perfusion through a rapid change of blood flow direction, and this phenomenon can be seen with a balloon test occlusion of the ICA and carotid clamping during carotid endarterectomy ${ }^{1,1,20)}$. Whereas, secondary collateral flow, such as from the leptomeningeal collaterals, needs more time for formation. Although all enrolled patients in this study had collateral MCA flow via the circle of Willis or an ophthalmic artery, there were discrepancies in baseline neurological deficits relative to ICA occlusion etiology. Atherosclerotic ICA occlusions were related to preceding intra/extracranial carotid stenosis, which could promote collateral flow ${ }^{177}$. Therefore, the atherosclerotic ICA occlusion group might be associated with a lower baseline NIHSS score.

Regarding the ICA occlusion etiology, 51.2\% of atherosclerotic ICA occlusions and $76.3 \%$ of non-atherosclerotic ICA occlusions received EVT. For this aspect of EVT, the EVT strategy was significantly different between the groups (Table 5). Additional procedures, such as balloon angioplasty and/or carotid stenting, were applied more frequently in the atherosclerotic occlusion group. Considering EVT strategy relative to the etiology of the ICA occlusion could be helpful toward achieving a better EVT outcome. Another concern is downstream distal embolization, which can cause L type ICA occlusions, T type ICA occlusions, or MCA occlusions. Therefore, a balloon guide catheter was applied during EVT for the prevention of distal embolization, except in four cases. Distal embolization occurred in 17 patients (33.3\%) during EVT, which occurred only in the non-atherosclerotic occlusion group. Although the distal embolization rate was high, mTICI $2 \mathrm{~b}$ or 3 of reperfusion was achieved in $75.9 \%$ of patients in the non-atherosclerotic occlusion group. This reperfusion rate is comparable to the result of meta-analysis, which showed an mTICI $2 \mathrm{~b}$ or 3 reperfusion rate of $71 \%^{8)}$. Furthermore, downstream distal embolization can occur regardless of EVT. Major neurological deterioration with an 8-point increase in NI- 
HSS score occurred for two patients (6.7\%) in the medical treatment group, which resulted in a carotid terminus and an MCA occlusion through spontaneous distal embolization. The mechanism might be similar with spontaneous recanalization in cardioembolic stroke. In the early period from index stroke, patients should be closely monitored for their neurological status.

However, this study has several limitations. First, this study excluded patients who did not receive TFCA due to minor neurological deficits and a low NIHSS score. Therefore, our results did not represent all patients with symptomatic ICA occlusion and collateral MCA flow. Second, it was retrospective study at a single center over a long period of data gathering. However, there were only two cases in the EVT group where modern thrombectomy devices, such as the Penumbra reperfusion catheter and the stent-retriever, were not available. So, even though the study inclusion period was long, the authors thought that the technical impact on EVT related to advances in devices would be minor.

\section{CONCLUSION}

In patients with symptomatic ICA occlusion and collateral MCA flow, decisions regarding treatment strategy based on the TFCA and stroke etiology can be helpful for achieving favorable clinical outcomes. EVT might be feasible for patients with atherosclerotic ICA occlusion and collateral MCA flow. For patients with a non-atherosclerotic ICA occlusion, EVT should be performed cautiously to prevent distal embolization for a better clinical outcome. Further prospective trials are needed to confirm the results of this study.

\section{CONFLICTS OF INTEREST}

No potential conflict of interest relevant to this article was reported.

\section{INFORMED CONSENT}

This type of study does not require informed consent.

\section{References}

1. American Society of Interventional and Therapeutic Neuroradiology : Carotid artery balloon test occlusion. AJNR Am J Neuroradiol 22(8 Suppl) : S8-S9, 2001

2. Barreto $A D$, Albright $K C$, Hallevi $H$, Grotta JC, Noser EA, Khaja AM, et al. : Thrombus burden is associated with clinical outcome after intraarterial therapy for acute ischemic stroke. Stroke 39 : 3231-3235, 2008

3. Berkhemer OA, Fransen PS, Beumer $D$, van den Berg LA, Lingsma HF, Yoo AJ, et al. : A randomized trial of intraarterial treatment for acute ischemic stroke. N Engl J Med 372 : 11-20, 2015

4. Bisschops RH, Klijn CJ, Kappelle LJ, van Huffelen AC, van der Grond J : Collateral flow and ischemic brain lesions in patients with unilateral carotid artery occlusion. Neurology $60: 1435-1441,2003$

5. Bradac GB, Venturi F, Stura G, Coriasco M, Ventilii G, Garabello D, et al. : Considerations about occlusion of the intracranial distal internal carotid artery. Clin Neuroradiol 27 : 169-174, 2017

6. Campbell BC, Mitchell PJ, Kleinig TJ, Dewey HM, Churilov L, Yassi N, et al. : Endovascular therapy for ischemic stroke with perfusion-imaging selection. N Engl J Med 372 : 1009-1018, 2015

7. Goyal M, Demchuk AM, Menon BK, Eesa M, Rempel JL, Thornton J, et al. : Randomized assessment of rapid endovascular treatment of ischemic stroke. N Engl J Med 372 : 1019-1030, 2015

8. Goyal M, Menon BK, van Zwam WH, Dippel DW, Mitchell PJ, Demchuk $A M$, et al. : Endovascular thrombectomy after large-vessel ischaemic stroke: a meta-analysis of individual patient data from five randomised trials. Lancet 387 : 1723-1731, 2016

9. Hacke W, Kaste M, Fieschi C, von Kummer R, Davalos A, Meier D, et al. : Randomised double-blind placebo-controlled trial of thrombolytic therapy with intravenous alteplase in acute ischaemic stroke (ECASS II). Second European-Australasian acute stroke study investigators. Lancet 352 : 1245-1251, 1998

10. Jauch EC, Saver JL, Adams HP Jr, Bruno A, Connors JJ, Demaerschalk $B M$, et al. : Guidelines for the early management of patients with acute ischemic stroke: a guideline for healthcare professionals from the american heart association/american stroke association. Stroke 44 : 870 947, 2013

11. Jovin TG, Chamorro A, Cobo E, de Miquel MA, Molina CA, Rovira A, et al. : Thrombectomy within 8 hours after symptom onset in ischemic stroke. N Engl J Med 372 : 2296-2306, 2015

12. Kikuchi K, Yoshiura T, Hiwatashi A, Togao O, Yamashita K, Honda H : Balloon test occlusion of internal carotid artery: angiographic findings predictive of results. World J Radiol 6 : 619-624, 2014

13. Kim JT, Park MS, Choi KH, Nam TS, Choi SM, Cho KH : Clinical implications of collateral middle cerebral artery flow in acute ischaemic stroke with internal carotid artery occlusion. Eur J Neurol 18 : 1384-1390, 2011

14. Lee SU, Hong JM, Kim SY, Bang OY, Demchuk AM, Lee JS : Differentiating carotid terminus occlusions into two distinct populations based on Willisian collateral status. J Stroke 18 : 179-186, 2016

15. Li W, Yin Q, Xu G, Liu X: Treatment strategies for acute ischemic stroke 
caused by carotid artery occlusion. Interv Neurol 5 : 148-156, 2016

16. Liebeskind DS : Collateral circulation. Stroke 34 : 2279-2284, 2003

17. Liebeskind DS, Cotsonis GA, Saver JL, Lynn MJ, Turan TN, Cloft HJ, et al. : Collaterals dramatically alter stroke risk in intracranial atherosclerosis. Ann Neurol 69 : 963-974, 2011

18. Liebeskind DS, Flint AC, Budzik RF, Xiang B, Smith WS, Duckwiler GR, et al. : Carotid I's, L's and T's: collaterals shape the outcome of intracranial carotid occlusion in acute ischemic stroke. J Neurolnterv Surg 7 : 402-407, 2015

19. Paciaroni M, Caso V, Venti M, Milia P, Kappelle LJ, Silvestrelli G, et al. : Outcome in patients with stroke associated with internal carotid artery occlusion. Cerebrovasc Dis 20 : 108-113, 2005

20. Sato $K$, Shimizu $H$, Inoue $T$, Fujimura $M$, Matsumoto $Y$, Kondo $R$, et al. : Angiographic circulation time and cerebral blood flow during bal-
Ioon test occlusion of the internal carotid artery. J Cereb Blood Flow Metab 34 : 136-143, 2014

21. Saver JL, Goyal M, Bonafe A, Diener HC, Levy El, Pereira VM, et al. : Stent-retriever thrombectomy after intravenous t-PA vs. t-PA alone in stroke. N Engl J Med 372 : 2285-2295, 2015

22. Saver JL, Jahan R, Levy El, Jovin TG, Baxter B, Nogueira RG, et al. : Solitaire flow restoration device versus the merci retriever in patients with acute ischaemic stroke (SWIFT): a randomised, parallel-group, noninferiority trial. Lancet 380 : 1241-1249, 2012

23. Zaidat O0, Yoo AJ, Khatri P, Tomsick TA, von Kummer R, Saver JL, et al. : Recommendations on angiographic revascularization grading standards for acute ischemic stroke: a consensus statement. Stroke 44 : 26502663, 2013 